\title{
Monitoreo en línea de accesorios de instalaciones eléctricas subterráneas mediante emisiones acústicas
}

\section{Online Monitoring of Accessories for Underground Electrical Installations through Acoustics Emissions}

\author{
Casals-Torrens P. \\ Departamento de Ingeniería Eléctrica \\ Universidad Politécnica de Cataluña, Barcelona, España \\ Correo:p.casals@upc.edu \\ González-Parada A. \\ Departamento de Ingeniería Eléctrica \\ División de Ingenierías Campus Irapuato-Salamanca \\ Universidad de Guanajuato \\ Correo:gonzaleza@salamanca.ugto.mx
}

\author{
Bosch-Tous R. \\ Departamento de Ingeniería Eléctrica \\ Universidad Politécnica de Cataluña,Barcelona, España \\ correo:bosch@ee.upc.edu
}

Información del artículo: recibido: septiembre de 2010, aceptado: marzo de 2011

\section{Resumen}

Las ondas acústicas provocadas por las descargas parciales en el interior de un dieléctrico pueden ser detectadas por sensores de Emisiones Acústicas (EA) y analizadas en el dominio del tiempo. Los resultados experimentales que se presentan, evidencian la capacidad de detección en línea de estos sensores, en el entorno próximo a un accesorio de cable, empalme o terminal. Los sensores EA son inmunes a las interferencias electromagnéticas, son un método de detección no destructivo y garantizan desacople galvánico respecto a la red eléctrica; esta técnica de detección de descargas parciales puede ser aplicada como método de prueba para mantenimiento preventivo o predictivo (mantenimiento basado en la condición), en equipos o instalaciones de media y alta tensión en servicio, y representa una alternativa a los sistemas eléctricos de detección, convencionales o no, que continúan basándose en la detección del impulsos de corriente. En el presente trabajo se presentan las pruebas de caracterización de los sensores EA por medio de pruebas comparativas de descargas parciales en accesorios para cables subterráneos de energía.

\section{Descriptores}

- cables de energía

- instalaciones subterráneas

- descargas parciales

- detección de fallas

- detección en línea

- detección acústica 


\begin{abstract}
The acoustic waves caused by Partial Discharges inside the dielectric materials, can be detected by acoustic emission (AE) sensors and analyzed in the time domain. The experimental results presented, show the online detection capability of these sensors in the environment near a cable accessory, such as a splice or terminal. The AE sensors are immune to electromagnetic interference and constitute a detection method non-intrusive and non-destructive, which ensures a galvanic decoupling with respect to electric networks, this technique of partial discharge detection can be applied as a test method for preventive or predictive maintenance (condition-based maintenance) to equipments or facilities of medium and high voltage in service and represents an alternative method to electrical detection systems, conventional or not, that continue to rely on the detection of current pulses. This paper presents characterization tests of the sensors AE through comparative tests of partial discharge on accessories for underground power cables.
\end{abstract}

\section{Introducción}

La fiabilidad y continuidad del suministro eléctrico en las redes subterráneas de distribución y transporte de energía eléctrica en alta tensión, se ven afectadas por la elevada tasa de falla de los accesorios empleados para su instalación, como son los empalmes y las terminales, en comparación con las fallas presentadas en los propios cables (Working Group, 1991; Bucci et al., 1994; Mashikian, 2000).

Esto esencialmente se debe a que finalizada la fabricación de los cables y antes de su instalación, estos son sometidos a rigurosos ensayos de calidad, tanto de rigidez dieléctrica, como de descargas parciales, que aseguran la ausencia de defectos de fabricación en los mismos. Los accesorios, en cambio, no pueden ser evaluados al 100\% como conjunto (Standard IEC 60840, 2004; Standard IEC 60502-4, 2005), ya que después de la fabricación y ensayo de sus partes, sufren un proceso de manipulación durante el montaje que también afecta la instalación del cable. Todo este proceso está propenso a errores y daños que difícilmente pueden ser detectados durante los ensayos de puesta en servicio de la instalación, debido a las limitaciones en las pruebas de campo, ya que son menos rigurosas que las pruebas de laboratorio (como el menor nivel de tensión, tiempos de prueba inferiores y menor sensibilidad en los equipos de detección de descargas parciales) y que en muchos casos, la prueba de descargas parciales no se exige (Standard IEC 60840, 2004) y normalmente tampoco se realiza, lo cual puede derivar en una mala instalación con la consecuente falla prematura, como es el caso de las fallas presentadas en las instalaciones subterráneas en el centro histórico de la Cd. de México.

En este sentido, toda nueva tecnología que pueda contribuir a garantizar la confiabilidad en el servicio y facilitar la obtención de la información, que permita con antelación, identificar potenciales situaciones de falla en los accesorios, sin duda reviste un especial interés, ya que permitirá efectuar de manera planificada los descargos y las operaciones preventivas de mantenimiento en las instalaciones, sin la urgencia, el costo económico y el consecuente desprestigio del servicio que provocan las interrupciones debidas a fallas "imprevistas".

Por otro lado, los métodos eléctricos ofrecen una alta confiabilidad cuando se usan en laboratorios de prueba apantallados o zonas libres de interferencias, pero en pruebas de campo resultan costosos y muy sensibles a las interferencias de Radio Frecuencia (RF); lo cual abre un espacio de oportunidades a sistemas no convencionales, no eléctricos, como el sistema propuesto en este trabajo, basado exclusivamente en EA, cuyos resultados se presentan.

\section{Detección de descargas parciales (DP)}

Existen diferentes métodos para la detección de las DP, los cuales están caracterizados en función del tipo de sensor que se emplea para la detección, estos se pueden clasificar en dos grandes grupos:

- Detección con sensores eléctricos (inductivo, capacitivo, inductivo-capacitivo).

- Detección con sensores acústicos.

\section{Detección con sensores eléctricos}

Los sistemas de detección de DP convencional sean normalizados (IEC) o no (Standard IEC 60885-3, 1988), se basan en la detección de impulsos de corriente y de tensión (Casals, 2008), los cuales presentan una serie de ventajas y desventajas que a continuación se presentan: 


\section{Desventajas:}

- En pruebas de campo son muy sensibles a las interferencias de RF, al no estar apantallados.

- Las interferencias son reducidas o eliminadas por sofisticados sistemas de discriminación (hardware o software), que pueden filtrar o cubrir las descargas.

- Los inductivos están limitados por el tipo constructivo de la pantalla del cable.

- La sensibilidad de los capacitivos está limitada por su bajo valor de capacidad de acoplamiento con respecto a la capacidad del objeto bajo ensayo (accesorio + cable).

- El tratamiento de la respuesta del sensor, en la mayoría de los casos, se realiza con equipos de medida de descargas parciales.

- Son costosos, del orden del medio centenar de miles de USD (equipo de detección más accesorios), lo cual limita su uso generalizado y su instalación permanente en campo.

Ventajas:

- Permiten conocer el nivel de descarga.

- Se pueden complementar con sistemas de localización de descargas.

- $\quad$ El uso de doble sensor permite auto calibración y analizar la dirección de descargas en empalmes.

- Existen sistemas comerciales para alta tensión (AT).

\section{Detección con sensores acústicos}

Con una metodología diferente, basada en la detección de variaciones de presión en la superficie del aislamiento se ofrece una solución al problema de detección de descargas parciales en accesorios, en línea y en campo, sin presentar las mismas desventajas de los sensores eléctricos (Casals, 2008), éstas se presentan a continuación:

Desventajas:

- Actualmente la mayoría de sensores y sistemas de detección están en desarrollo.

- No se comercializan equipos para aplicaciones específicas en accesorios de cables de AT.

- Reducción de sensibilidad, debido a la atenuación con la distancia y con las elevadas temperaturas que pueden alcanzar el material de aislamiento.

- Los detectores de uso externo en cables de Media Tensión (MT), tienen una sensibilidad limitada por las interfases de aire (baja transmisión acústica) que hay en el interior de los cables o empalmes.

- No permiten conocer el nivel de descarga.

- La calibración es compleja.

Ventajas:

- Insensibles a las interferencias de Radio Frecuencia $(\mathrm{RF})$, no requieren pantalla.

- Insensibles a los campos electromagnéticos.

- Es un método de ensayo no destructivo.

- Sensores de alta sensibilidad, sólo requieren amplificación y visualización con osciloscopio digital, sin tratamientos complejos de señal.

- Tienen amplio espectro de frecuencia.

- El tipo constructivo de pantalla del cable no impide su aplicación.

- Alta robustez mecánica.

- Alta resistividad eléctrica que ofrece separación galvánica respecto al equipo ensayado.

- Pueden ofrecer precios de comercialización de un orden de magnitud 10 veces menor, (con sensores y osciloscopio de medición), lo cual permite un uso generalizado y su instalación unitaria por empalme.

\section{Conceptos de detección acústica de Descargas Parciales (DP)}

La DP en el interior de un sistema aislante representa una pequeña explosión con colisiones entre moléculas, la cual excita la emisión de ondas de presión acústica (Harrold, 1986; Kreuger, 1991; Lundgaard, 1992), que se propagan a través del material del aislamiento. Estas ondas mecánicas pueden ser detectadas por sensores de EA, sensibles a los cambios de presión que se producen en la superficie del aislamiento y que envuelve la cavidad o defecto, especialmente en las capas finas semiconductoras.

La energía que transfiere una descarga, en un defecto tipo cavidad, se puede calcular por la diferencia entre la energía almacenada antes y después de la descarga parcial. La magnitud de la energía liberada $(W)$ en Joule, expresada en función del valor eficaz de la tensión de excitación de la descarga ( $V i$ ) en volts, a una carga eléctrica $q_{c}$ en Coulombs, que es transferida cuando se presenta una descarga en un defecto tipo cavidad se puede expresar como:

$\mathrm{W} \cong(1 / \sqrt{2}) \cdot \mathrm{q}_{\mathrm{c}} \cdot \mathrm{V}_{\mathrm{i}(\mathrm{rms})}=0,707 \cdot \mathrm{q}_{\mathrm{c}} \cdot \mathrm{V}_{\mathrm{i}(\mathrm{rms})}$

Esta energía puede presentar órdenes de magnitud de $10^{-9}$ a $10^{-5} \mathrm{~J}$ (Naidu y Kamaraju, 1995; Mason, 1995) y va- 
ría con el espesor de aislamiento, provocando localmente diferentes efectos, entre ellos, la radiación de energía en forma de ondas acústicas.

Con el fin de verificar la cantidad de energía presente durante la descarga en una cavidad del tipo esférico, se realizó una simulación por medio de elementos finitos de una cavidad de $1 \mathrm{~mm}$ de diámetro en el interior de un aislamiento de $18 \mathrm{~mm}$ de espesor de un cable de energía de alta tensión, con aislamiento de Polietileno de cadena cruzada (XLPE), con un conductor de aluminio de $1 \times 400 \mathrm{~mm}^{2}$, para una tensión de 76/132 kV. En la figura 1 se muestra el resultado obtenido de la simulación, en donde se aprecia el incremento del gradiente de tensión en la cavidad cercana a la pantalla semiconductora interior, encontrándose que esta energía, antes de que se produzca una descarga parcial, cuando el cable tiene una tensión de $76 \mathrm{kV}$, se encuentra en un rango que varía de $25,6 \times 10^{-5} \mathrm{~J}$ a $5,59 \times 10^{-5} \mathrm{~J}$, entre la zona de máximo gradiente y mínimo gradiente, respectivamente (Casals, 2008).

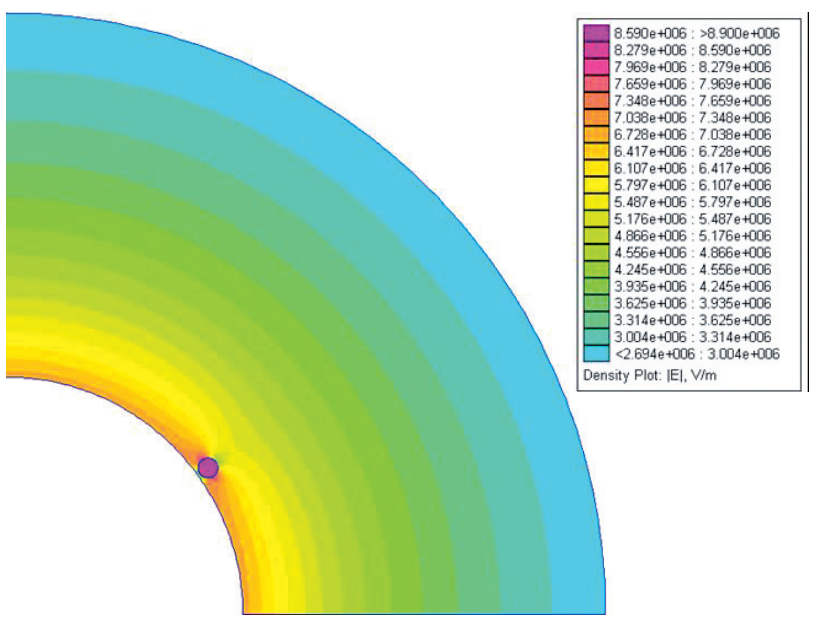

Figura 1. Simulación por elemento finito de una cavidad adyacente a la capa semiconductora interior

El sonido de la descarga se propaga a través de un medio de densidad $(\rho)$ en $\mathrm{kg} / \mathrm{m}^{3}$, a una velocidad $(c) \mathrm{m} / \mathrm{s}$, de forma mecánica, mediante el movimiento de ondas longitudinales que provocan cambios locales de presión $(p)$ en Pascals y desplazamiento de las moléculas del medio. La ecuación de propagación de onda para campos de presión esféricamente simétricos se representa en la ecuación 2 (Kinsler et al., 2004; Blackstock, 2000) :

$\nabla^{2} p=\frac{1}{c^{2}} \frac{\partial^{2} p}{\partial t^{2}}$
Los pulsos de descargas parciales tienen duraciones muy cortas, que oscilan desde $1 \mathrm{~ns}$ a cientos de ns (Harrold, 1986; Naidu y Kamaraju, 1995; Boogs, 1990), esto provoca que el espectro de frecuencia de las ondas acústicas generadas sea muy amplio en el rango de los ultrasonidos, los cuales pueden ser del orden de $\mathrm{kHz}$ hasta los $\mathrm{MHz}$.

Si consideramos la descarga parcial en una cavidad como una fuente puntual simple, generalmente muy pequeña e inferior a $1 \mu^{3}$; cuando inicia su actividad emite ondas acústicas esféricas de simetría radial, como se muestra en la figura 2.

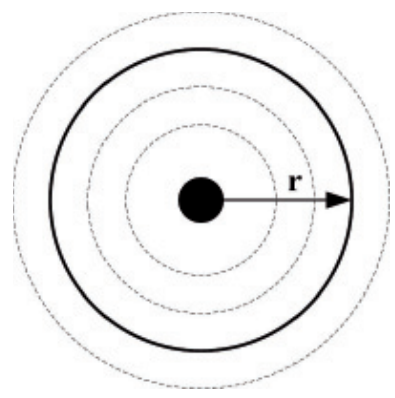

Figura 2. Propagación de ondas acústicas de fuente puntual

En el interior del empalme en un cable, la descarga parcial se produce siguiendo la dirección radial de máxima variación del campo eléctrico. Esta misma dirección es la más corta en la propagación de las ondas acústicas esféricas, hasta la frontera con un medio diferente al aislamiento, de acuerdo con la representación de la figura 3, donde $(r)$ representa el radio que alcanza en $\mathrm{m}$.

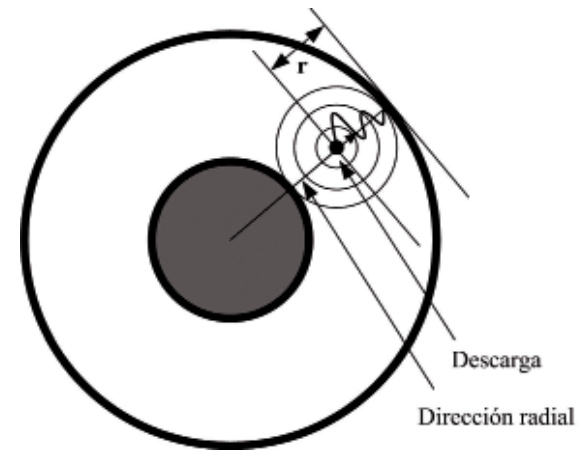

Figura 3. Representación de la propagación radial de una onda acústica

En consecuencia, lo primero que alcanzan los frentes de presión de las ondas acústicas son ambas capas semiconductoras y el conductor, con una diferencia de tiempo que dependerá de la posición de la descarga en el interior del aislamiento. Entre estos medios se produce 
la refracción o reflexión de dicha onda, en función de la impedancia acústica específica $(\vec{z})$ de los mismos, la cual se define como la razón de la presión acústica del medio $(\vec{p})$ en Pascals y la velocidad de partícula asociada $(\vec{v})$ en $\mathrm{m} / \mathrm{s}$ :

$\overrightarrow{\mathrm{z}}=\frac{\overrightarrow{\mathrm{p}}}{\overrightarrow{\mathrm{V}}}$

que también puede expresarse como:

$\vec{z}=\rho_{o} c \cos \beta e^{\mathrm{j} \beta}$

donde $\left(\rho_{o}\right)$ es la densidad característica del material en $\mathrm{kg} / \mathrm{m}^{3}$ y $\beta$ el ángulo entre la dirección de la presión acústica y la velocidad de partícula en el material. $\beta=0$ cuando la onda es plana.

Los frentes de ondas que siguen direcciones no radiales, como la dirección axial del cable (figura 4), recorren un camino más largo, pero finalmente también alcanzan e inciden oblicuamente sobre las capas semiconductoras y el conductor.

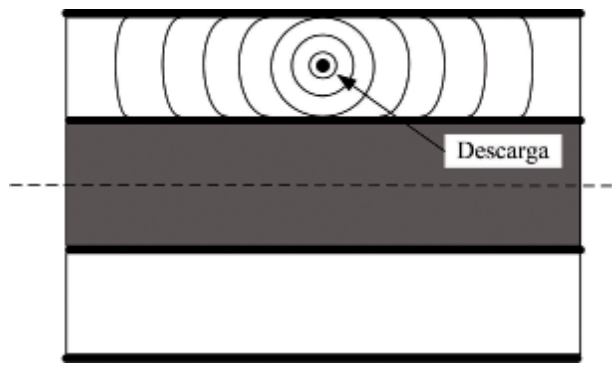

Figura 4. Representación de la propagación axial de ondas acústicas

La velocidad longitudinal del sonido en el polietileno se incrementa linealmente con la densidad y puede variar aproximadamente entre 2003 m/s y 1977 m/s (Piche et al., 1992), dependiendo del grado de reticulación del material.

En la mayoría de polímeros, comúnmente usados en aislamientos de media y alta tensión, el coeficiente de atenuación se reduce aproximadamente de forma lineal con el incremento del módulo de Young del material (E) (Harrold, 1986). En la ecuación 5 se muestra el módulo de Young en función del grado de rigidez del material, y su magnitud disminuye con el incremento de temperatura (Suzuki et al., 1988).

$\mathrm{E}=\rho \cdot \mathrm{c}^{2}$

La incidencia de la atenuación es el condicionante que limita el uso de esta técnica a detecciones en cortas distancias, pero a su vez, la hace adecuada para el control focalizado en equipos de poca longitud, como los accesorios de cables.

\section{Evaluación del sistema de detección acústica}

Para la evaluación del comportamiento de un sistema de detección acústica en una instalación de cable de energía subterránea se empleó un transformador de prueba de alta tensión e instalaciones de cables de energía para media y alta tensión, incorporando sensores de Emisión Acústica (EA), externos y próximos al accesorio del cable, tal como se observa en la figuras 5 .

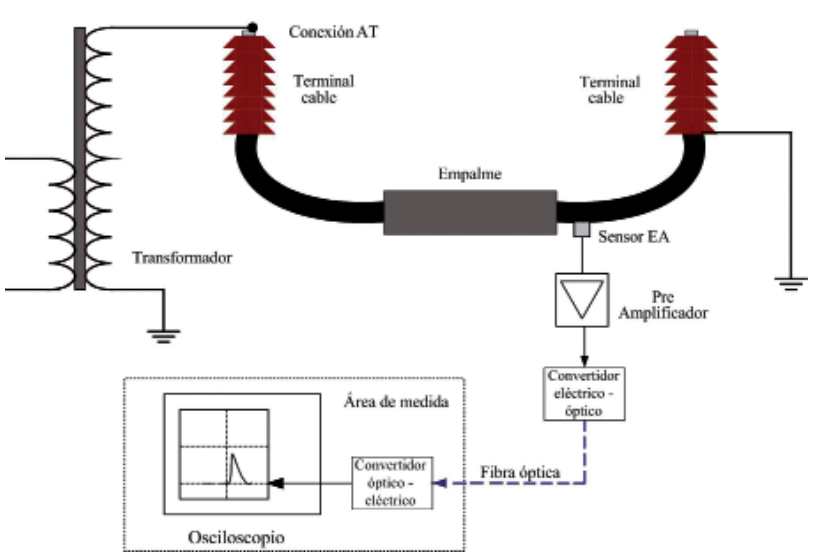

Figura 5. Esquema de prueba del sistema de detección acústico con un sensor colocado próximo a un empalme

El sensor o sensores, se recomiendan uno en cada extremo, activados por las ondas acústicas generan una señal de respuesta que se envía a un pre amplificador donde pueden combinarse las diferentes señales, tal como se muestra en la figura 6 .

Los sensores EA, ofrecen la sensibilidad para asegurar una información permanente (sistema en línea) o periódica (lectura según plan preventivo) de la actividad de DP detectada y en especial, de las variaciones de la misma en el tiempo, pudiendo generar alarmas según los diferentes niveles de actividad, como consecuencia de la degradación del accesorio.

En la figura 7 se muestra la ubicación del sensor EA en la cercanía de un empalme para alta tensión, en donde los componentes del sistema son: el sensor EA, un pre amplificador de banda ancha de $20 \mathrm{kHz}$ a $5000 \mathrm{kHz}$, convertidor electro óptico, salida por fibra óptica y convertidor óptico-eléctrico.

El equipo necesario para medida o análisis es un osciloscopio digital o un analizador de espectro. Se utilizó un osciloscopio de $200 \mathrm{MHz}$ y 1GSa/s, pero con un osciloscopio de mayores prestaciones se mejora la capacidad de detección. 


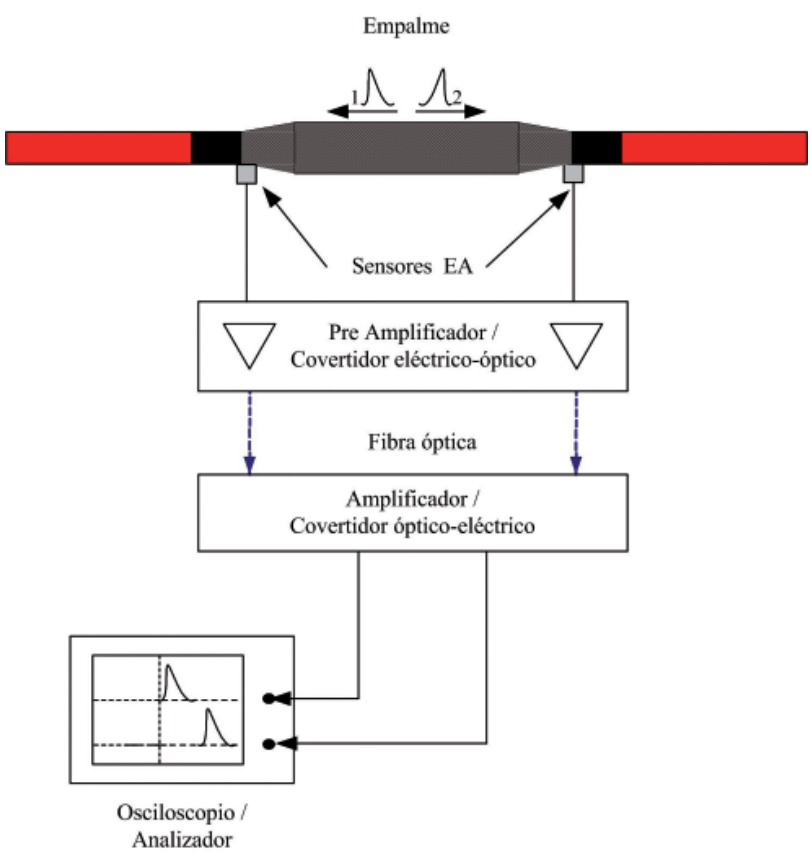

Figura 6. Esquema del sistema de detección acústico con dos sensores colocados al interior de un empalme

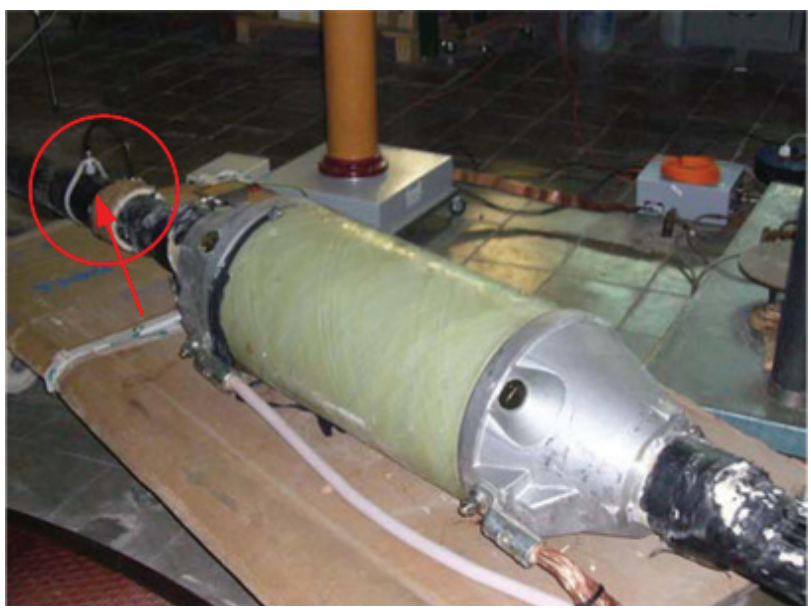

Figura 7. Sensor ubicado próximo a un empalme de alta tensión

Dada la importancia que tiene la seguridad para el técnico que realiza ensayos y mediciones en alta tensión, ésta se asegura con la elevada resistividad del sensor y la conexión mediante fibra óptica, garantizando además el desacople galvánico entre el sensor y el equipo de medida, considerando que la fibra óptica también permite alejar este último del accesorio bajo ensayo.

\section{Resultados experimentales}

Las detecciones de las DP se realizaron conjuntamente de forma eléctrica por medio de un detector convencio- nal de DP y por medio de sensores EA. La detección eléctrica nos permitió obtener las magnitudes de descarga en $\mathrm{pC}$ y verificar que el sistema acústico detecta la presencia de las DP, en la figura 8 se muestran los resultados de ambos sistemas de detección.

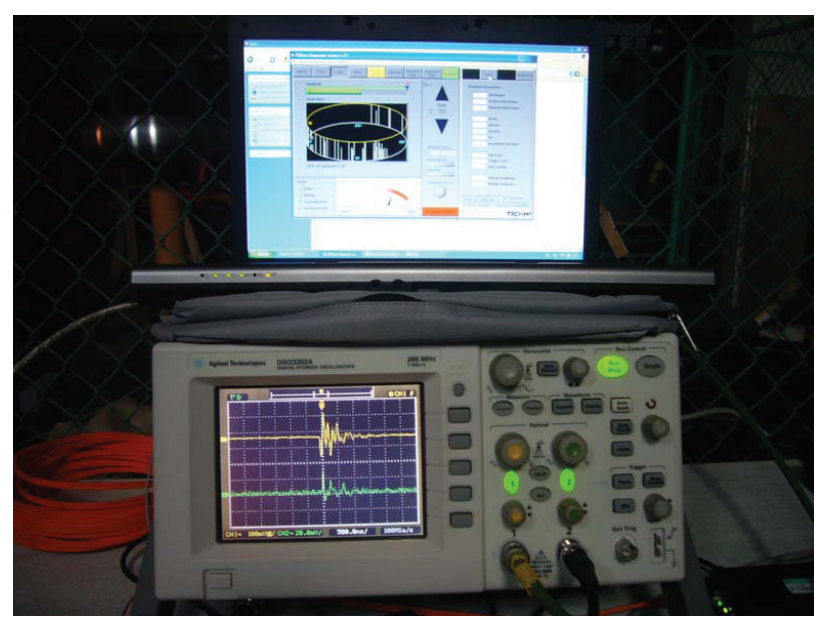

Figura 8. Detección eléctrica y acústica de DP

Con el fin de caracterizar cada una de las señales de DP, se utilizaron defectos típicos de cable o preparación de accesorios, como restos de material semiconductor, cortes en el semiconductor externo y huecos en el aislamiento, provocados durante la preparación del empalme, con el fin de obtener de manera artificial las descargas parciales. Estos defectos fueron realizados en dos tipos de cables, uno de ellos fue para alta tensión, de XLPE 76/132 kV 1x800 mm² Al y el otro para media tensión, de XLPE 12/20 kV 1x240 mm² Al.

La señal característica de cada uno de estos defectos se presenta en la figura 9, conjuntamente con la respuesta acústica obtenida y la correspondiente transformada rápida de Fourier.

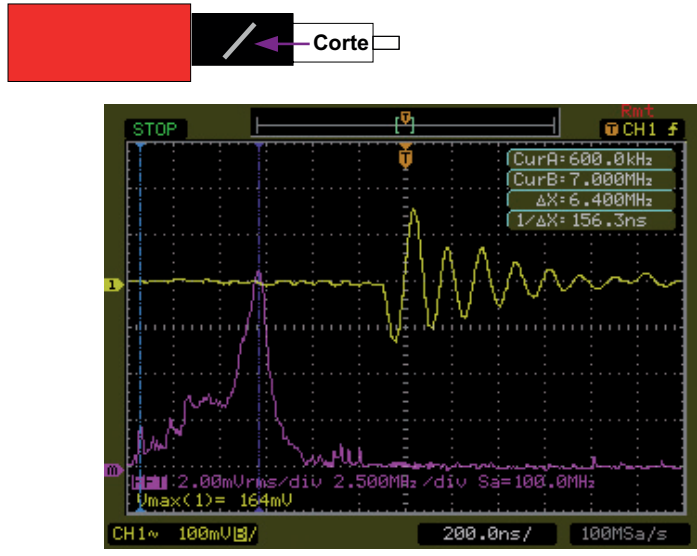

(a) Corte en el semiconductor - cable AT 

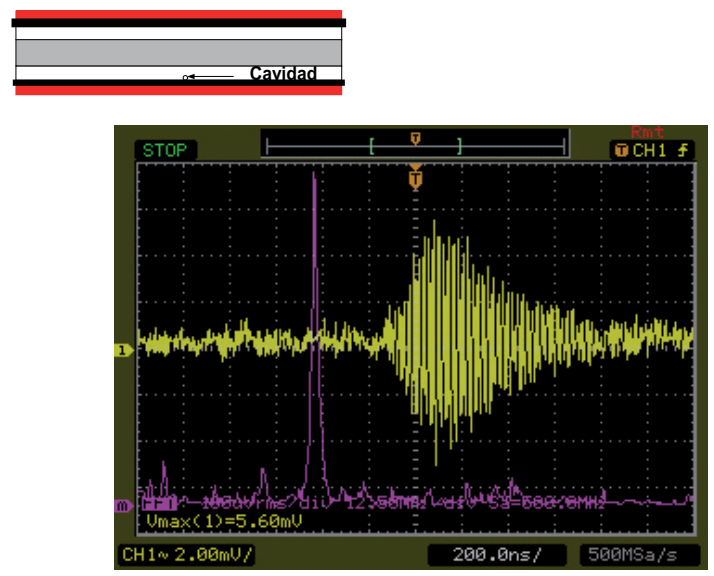

(b) Cavidad $\varnothing=2 \mathrm{~mm}$ en aislamiento - cable MT
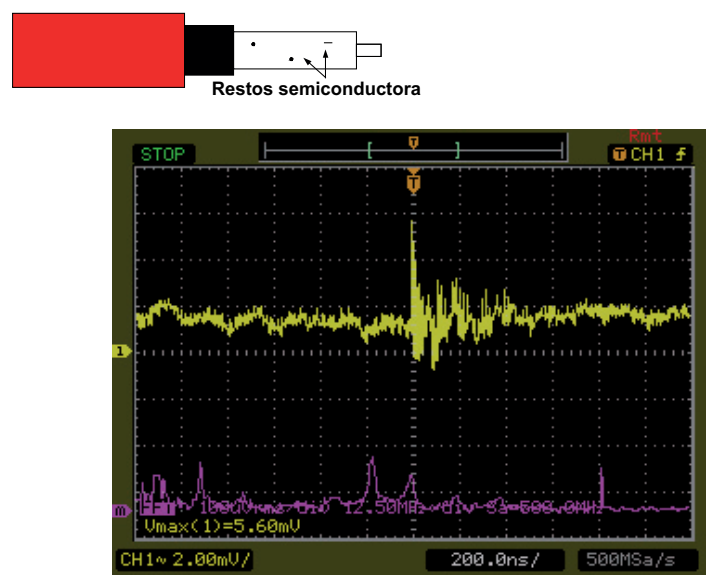

(c) Restos de semiconductor - cable MT

Figura 9. Modelos de descarga parcial

En la tabla 1 se resumen los resultados para los tres tipos de defectos y la ubicación del sensor EA respecto a cada una de las fallas presentadas.

\section{Conclusiones}

La evolución de los sensores EA se presenta como un refuerzo o alternativa a los sistemas eléctricos, en la detección de DP en accesorios de cables, para aplicaciones en línea y en campo.

El sistema presenta mayor sensibilidad ante descargas internas del aislamiento, tipo cavidad, contenidas bajo el semiconductor.

El nivel de sensibilidad alcanzado, descargas a partir de $40 \mathrm{pC}$, pueden ser una alternativa en aplicaciones de $\mathrm{MT}$, pero requiere mejorarse para AT.

La respuesta de los sensores EA, ofrece diferentes formas ante diferentes tipos de defecto, lo cual da pie a continuar el trabajo con el análisis detallado de la transformada rápida de Fourier (FFT), que también reflejan estas diferencias.

\section{Referencias}

Blackstock D.T. Fundamentals of Physical Acoustic, New York,WileyInterscience, 2000.

Boogs S.A. Partial Discharge: Overview and Signal Generation. IEEE Electrical Insulation Magazine, 6(4):33-39, julio-agosto, 1990.

Bucci R.M., Rebbapragada R.V., McElroy A.J., Chebli E.A., Driller S. Failure Prediction on Underground Distribution Feeder Cables. IEEE Transactions on Power Delivery, 9(4):D2.1-D2.7, octubre 1994.

Casals-Torrens P. Sistema en línea para detección preventiva de descargas parciales en accesorios de cables de alta tensión mediante sensores de emisiones acústicas. Tesis (doctoral). Barcelona, Universitat Politècnica de Catalunya, Dpto. de Ingeniería Eléctrica, 2008.

Harrold R.T. Acoustic Theory Applied to the Physics of Electrical Breakdown in Dielectrics. IEEE Transactions on Electrical Insulation, EI-21(5):781-792, octubre 1986.

\begin{tabular}{ccccc}
\hline $\begin{array}{c}\text { Tipo } \\
\text { Defecto }\end{array}$ & $\begin{array}{c}\text { Tensión } \\
(\mathrm{kV})\end{array}$ & $\begin{array}{c}\text { Medida DP } \\
(\mathrm{pC})\end{array}$ & $\begin{array}{c}\text { Medida } \\
\text { acústica }(\mathrm{mV})\end{array}$ & $\begin{array}{c}\text { Ubicación } \\
\text { del sensor }\end{array}$ \\
\hline a & 70 & 40 & 136 & Sobre semicon. a 25cm del defecto \\
a & 70 & 80 & 164 & Sobre semicon. a 50cm del defecto \\
b & 12 & 40 & 3,68 & Sobre semicon. a 50cm del defecto \\
b & 14 & 50 & 5,6 & Sobre semicon. a 50cm del defecto \\
b & 17 & 70 & 10 & Sobre cubierta a 50cm del defecto \\
c & 8,4 & 60 & 5,6 & Sobre semicon. a 10cm del defecto \\
c & 8,2 & 64 & No detecta & Sobre semicon. a 90 cm del defecto \\
c & 16,2 & 80 & 2 & Sobre semicon. a 90 cm del defecto \\
\hline
\end{tabular}

Tabla 1. Medidas obtenidas dependiendo del defecto en el accesorio 
Kinsler L.E, Frey A.R., Coppens A.B., Sanders J.V. Fundamentals of Acoustic, 4a ed., New York, John Wiley \& Sons, Inc, 2004.

Kreuger F.H. Industrial High Voltage, Editorial Delf University Press, Delf-Netherlands, 1991.

Lundgaard L.E. Partial Discharge-Part XII. Acoustic Partial Discharge Detection-Fundamental Considerations. IEEE Electrical Insulation Magazine, 8(4):25- 31, julio-agosto 1992.

Mashikian M.S. Partial Discharge Location as a Diagnostic Tool for Power Cables. Power Engineering Society Winter Meeting, 2000. IEEE, 3:1604-1608, January 2000.

Mason J.H. Enhancing the Significance of PD Measurements. IEEE Transactions on Dielectrics and Electrical Insulation, 2(5):876-888, octubre 1995.

Naidu M.S., Kamaraju V.High Voltage Engineering, 2a ed., USA, Mc Graw Hill, 1995.

Piche L., Pelissou S., Crine J.P. Cable Insulation Density Profile Determined from Ultrasonic Velocity Measurements. Electrical Insulation Magazine, IEEE, 8(3):33-36, mayo-junio 1992.
Standard IEC 60840: 2004. Power Cables with Extruded Insulation and their Accessories for Rated Voltages Above $30 \mathrm{kV}$ (Um=36 $\mathrm{kV})$ up to $150 \mathrm{kV}(\mathrm{Um}=170 \mathrm{kV})$-Test Methods and Requirements.

Standard IEC 60502-4: 2005. Power Cables with Extruded Insulation and their Accessories for Rated Voltages from $1 \mathrm{kV}$ (Um= $1,2 \mathrm{kV})$ up to $30 \mathrm{kV}(\mathrm{Um}=36 \mathrm{kV})$. Part 4: Test Requirements on Accessories for Cables with Rated Voltages from $6 \mathrm{kV}$ (Um= $7,2 \mathrm{kV})$ up to $30 \mathrm{kV}(\mathrm{Um}=36 \mathrm{kV})$.

Standard IEC 60885-3: 1988. Electrical Test Methods for Electric Cables. Part 3: Test Methods for Partial Discharge Measurements on Lengths of Extruded Power Cables.

Suzuki H., Kanaoka, M., Sekii Y. Measurements on the Frozen Strain in XLPE Insulated Cables Using Thermo Mechanical Analysis, en: Proceedings of the Twenty-First Symposium on Electrical Insulating Materials, septiembre,1988, 26, pp. 255-258.

Working Grup 21.10. Survey on the Service Perfomance on HV AC Cable Systems. Report of Study Committee 21 Insulated Cables. Electra, (137):47-59, agosto 1991.

\section{Semblanza de autores}

Pau Casals-Torrens. Doctor en ingeniería industrial por la Universidad Politécnica de Cataluña (España, 2007). Ingeniero electricista por la Universidad de Oriente (Venezuela, 1984), realizó estudios de maestría en sistemas de potencia eléctrica en la Universidad Central de Venezuela, 1989. Ese mismo año ingresó como ingeniero en el departamento de calidad y ensayos eléctricos de la empresa BICC-General Cable, Manlleu, España. Desde 2001 es profesor del Departamento de Ingeniería Eléctrica de la Universidad Politécnica de Cataluña. Combina sus actividades docentes y de investigación con la participación en proyectos con empresas del sector eléctrico.

Adrián González-Parada. Ingeniero eléctrico por el Instituto Tecnológico de Querétaro, realizó estudios de maestría en ingeniería eléctrica con especialidad en alta tensión en la F.I.M.E.E. de la Universidad de Guanajuato y obtuvo el doctorado en ingeniería eléctrica por la Universidad Politécnica de Cataluña en Barcelona, España. Es profesor-investigador en la DICIS de la Universidad de Guanajuato y responsable del laboratorio de máquinas eléctricas y dispositivos especiales. Sus áreas de interés son la superconductividad aplicada en equipos eléctricos, el uso eficiente de la energía en los sistemas eléctricos y la confiabilidad de los equipos eléctricos en la red eléctrica.

Ricard Bosch-Tous. Doctor en ingeniería industrial por la Universidad Politécnica de Cataluña. Cuenta con 15 años de experiencia industrial en el ámbito de mantenimiento de equipos con mando electrónico de potencia en industria siderúrgica y automotriz. Desde 1984 es profesor del Departamento de Ingeniería Eléctrica de la ETSEIB-UPC. Actualmente dirige el laboratorio de alta tensión, alternadores y máquinas eléctricas especiales de la ETSEIB-UPC, su trabajo se centra en la optimización de toda la cadena energética en sistemas que utilicen electricidad. 